\title{
CORRIGENDUM
}

\section{Direct-access genetic testing: the view from Europe}

\section{Larry J. Kricka, Paolo Fortina, Yuan Mai and George P. Patrinos}

Nature Reviews Genetics 12, 670 (2011)

Two sentences in the above article contained incorrect information.

The sentence citing the study in reference 8 incorrectly stated that the study had been conducted through Facebook. The survey was in fact conducted online - but not through Facebook - so the sentence should have read "An online survey of $>1,000$ DTC customers in the United States found that most were satisfied with the testing experience and found their test results easy to understand".

Additionally, the sentence citing the survey conducted in reference 11 incorrectly stated that few respondents were willing to pay for direct-to-consumer (DTC) genetic testing. In fact, this study did not look specifically at DTC testing. The authors of the study concluded that few participants were willing to pay more than 500 Canadian dollars for genetic testing, so the sentence should have read "By contrast, a recent Canadian survey indicated that few respondents were willing to pay more than 500 Canadian dollars for genetic testing".

The article has been corrected online. The authors apologize for these errors. 